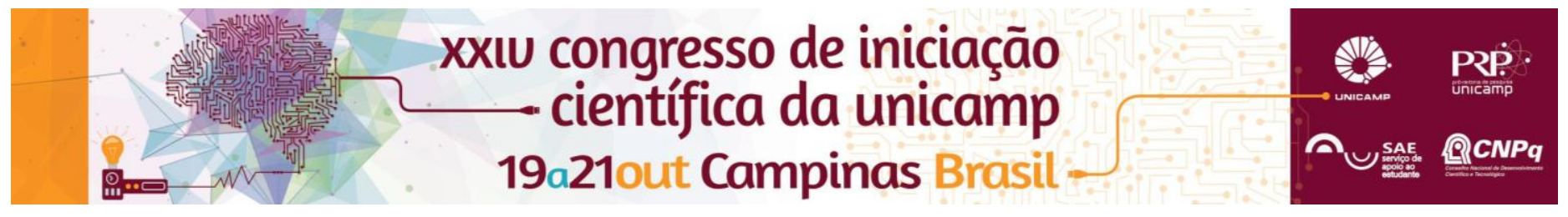

\title{
Design and Simulation of the Tissue Spheroids Fusion Process for Bioprinting
}

Viviane F. Lara*, Julia A. Nogueira, Rodrigo A. Rezende, Jorge Vicente L. da Silva.

\section{Abstract}

This work focuses on the study of the fusion of spheroids process used in bioprinting of human tissues and organs such as building blocks, through computer simulations.

\section{Key words:}

Tissue Spheroids, CFD computer simulations, Bioprinting.

\section{Introduction}

Organ printing can be defined as layer-by-layer additive robotic biofabrication of three-dimensional functional living macrotissues and organ constructs using tissue spheroids as building blocks. The microtissues and tissue spheroids are living materials with certain measurable, evolving and potentially controllable composition, material and biological properties.

In the produced model, the entry of water by needles in a structure consisting of alginate spheres was simulated.
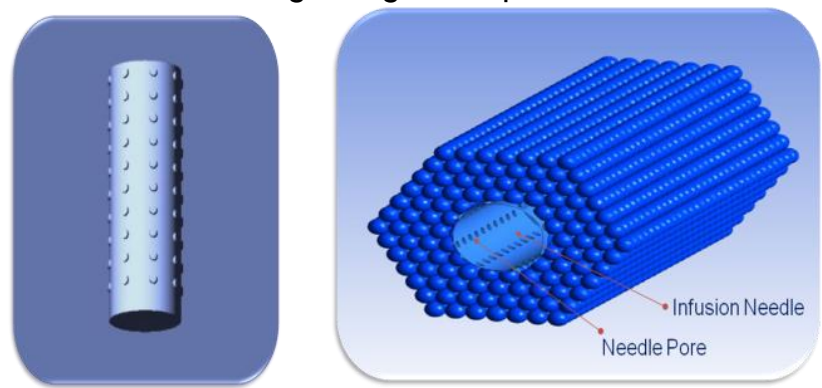

Image 1. Needle for perfusion (left) and a bulk of spheroids perfused by a needle (right).

\section{Results and Discussion}

After generating the design, simulations were performed in the ANSYS / CFX software. In the produced model was simulated entry of water by needles in a structure consisting of alginate spheres. Structures were made with overlapping layers and structures with layers rotated of 30 degrees each other to the analysis of the fluid insertion in the spheroids, inside and out of them, considering the fusion process.

The figures below represent the passage of water in an alginate structure, and the range of red to blue respectively represent water and alginate.

Speed: $5 \mathrm{~mm} / \mathrm{s}$; Total time: 10s.

Internal Flow to the fusion of $1 \%$ and $40 \%$ between spheroids:
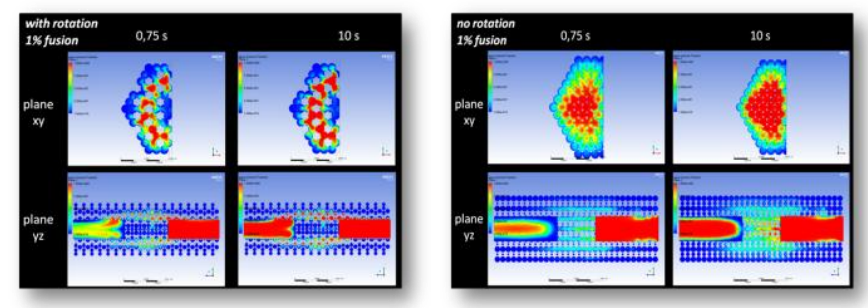

Image 2. Internal flow of water in the spheroids under $1 \%$ fusion (with rotation between the layers (left) and without rotation (right)).
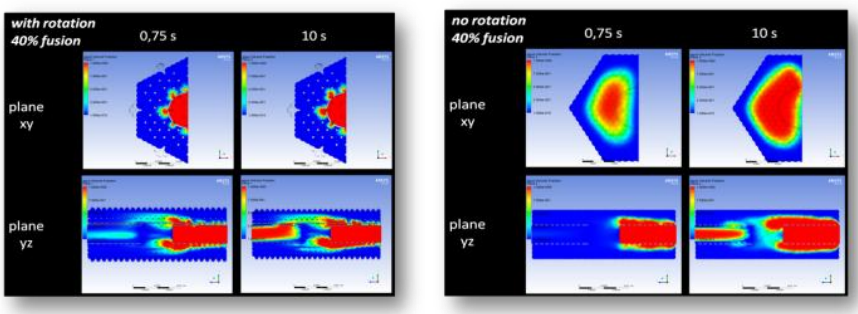

Image 3. Water flow in the spheroids on $40 \%$ fusion (with rotation between the layers (left) and without rotation (right)).

External flow to the spheroids under $1 \%$ and $20 \%$ fusions respectively :
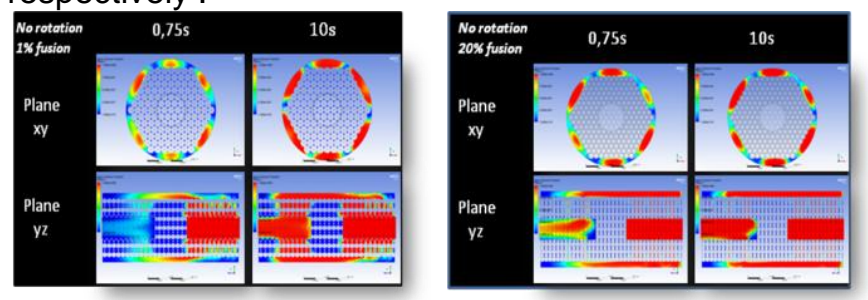

Image 4. Water flow in the spheroids without rotation and under $1 \%$ (left) and $20 \%$ (right) of fusion.

\section{Conclusions}

It was observed in the flow passage, inside the spheroids, that as the percentage of fusion increased, the water flow does not follow a standard and that this was probably due to the passage of alginate together with water.

After, in the analysis of water flow out of the spheroids, it was observed that as had increased fusion, seen by the images in the $y z$ plane, the passage of water was decreased.

\section{Acknowledgement}

My thanks to the CNPq/PIBIC program at CTI Renato Archer.

Nogueira, J. A.; Lara, V. F. ; Marques, T. S. ; Oliveira, D. S.; Mironov, V.; da Silva, J. V. L.; Rezende, R. A.. Simulation of a 3D bioprinted human vascular segment.

Computer Aided Chemical Engineering. 1ed.London: Elsevier, 2015, 37, $683-$ 688

Rezende, R.A.; Nogueira, J.A. ; Lara, V. F. ; Mironov, V. ; Maciel Filho, R.; da Silva, J.V.L. . Development of a Bioreactor by Computer Fluid Dynamics Simulations for the Maturation of 3D Printed Organs by Rapid Prototyping. Chemical Engineering Transactions, 2013, 32, 1153-1158. 